\title{
Imagem anular em tomografia computadorizada do tórax de alta resolução"
}

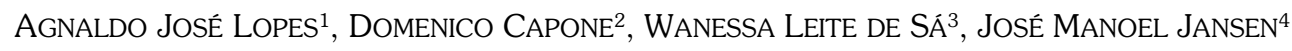

Descritores - Sarcoidose. Sarcoidose pulmonar. Tomografia computadorizada por raios X.

Key words - Sarcoidosis. Pulmonary sarcoidosis. X-ray computed tomography.

\section{APRESENTAÇÃO CLÍNICA}

Homem pardo, 32 anos, casado, instalador de aparelho de som, natural e residente no Rio de Janeiro, procurou atendimento médico queixando-se de tosse seca com um ano de evolução. Negava outros sintomas respiratórios, emagrecimento ou febre. Referia artralgia simétrica e cumulativa em cotovelos, punhos e tornozelos, sem sinais flogísticos associados. Negava tabagismo. Ao exame físico encontrava-se em bom estado geral e sem anormalidades.

Exames de imagem - A radiografia simples de tórax mostrava linfonodomegalia hilar bilateral (Figura 1). A tomografia computadorizada do tórax de alta resolução (TCAR) evidenciava aumento dos linfonodos hilares, micronódulos difusos e imagem anular de cerca de $4 \mathrm{~cm}$ de diâmetro, com parênquima normal de permeio, em lobo superior direito (Figuras 2 e 3).

Outros exames - Hematócrito $=51 \%$, hemoglobina $=$ $17 \mathrm{~g} / \mathrm{dl}$, leucócitos $=3.800 / \mathrm{mm}^{3}$. PPD não reator e dosagens de cálcio sérico e urinário normais. Exame de fundo de olho: edema cistóide em olho direito. A broncoscopia não evidenciava alterações de vias aéreas. A espirometria mostrava distúrbio ventilatório obstrutivo moderado. As medidas dos volumes pulmonares e da difusão do

\footnotetext{
Trabalho realizado no Hospital Universitário Pedro Ernesto.

1. Médico do Serviço de Pneumologia e Mestre em Pneumologia pela Universidade Federal Fluminense.

2. Professor Assistente do Serviço de Pneumologia do Hospital Universitário Pedro Ernesto e Radiologista do Hospital Geral de Bonsucesso.

3. Médica Estagiária do Serviço de Pneumologia.

4. Professor Titular e Chefe do Serviço de Pneumologia.

Endereço para correspondência - Agnaldo José Lopes, Rua José do Patrocínio, 290, Apto. 405 - 20560-160 - Rio de Janeiro, RJ. Tels. (21) 587-6357/587-6537/576-2532.
}

Recebido para publicação em 15/9/00. Aprovado, após revisão, em 12/3/01. monóxido de carbono foram normais. O exame anatomopatológico de fragmento retirado pela biópsia transbrônquica mostrou processo inflamatório crônico granulomatoso não caseoso com pesquisa negativa para micobactérias e fungos.

Diagnóstico - Sarcoidose gânglio-pulmonar.

\section{DISCUSSÃO}

Desde o seu surgimento, a tomografia computadorizada do tórax de alta resolução (TCAR) foi reconhecida como uma ferramenta importante na avaliação da sarcoidose pulmonar. Além de ver o pulmão com um detalhamento antes só possivel na observação microscópica da peça, pode-se estabelecer uma relação sinal (tomográfico) x significado (histológico), o que permitiu um grande avanço na busca diagnóstica e no acompanhamento das doenças pulmonares intersticiais.

A linfonodomegalia é tão comum quanto a doença do parênquima pulmonar em pacientes com sarcoidose. A tomografia computadorizada é superior à radiografia de tórax para definir a extensão do envolvimento ganglionar e demonstrar a sua presença em localizações atípicas tais como o mediastino anterior, axilas, a região subcarinal e a cadeia mamária interna. $\mathrm{O}$ acometimento linfonodal geralmente é simétrico e bilateral, e os gânglios linfáticos podem apresentar calcificações, inclusive com aspecto em "casca de ovo"(1).

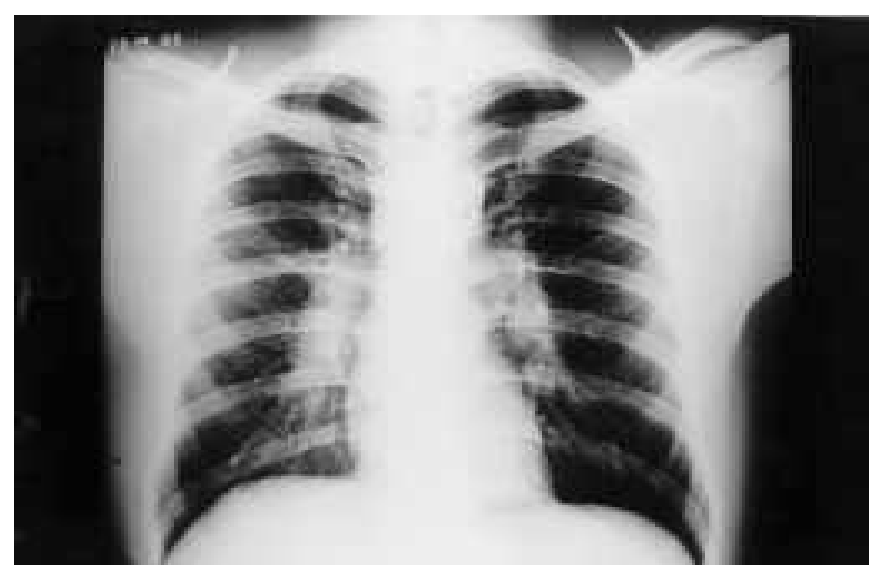

Figura 1 - Radiografia de tórax mostrando linfonodomegalia hilar bilateral e ausência de anormalidades no parênquima pulmonar. 

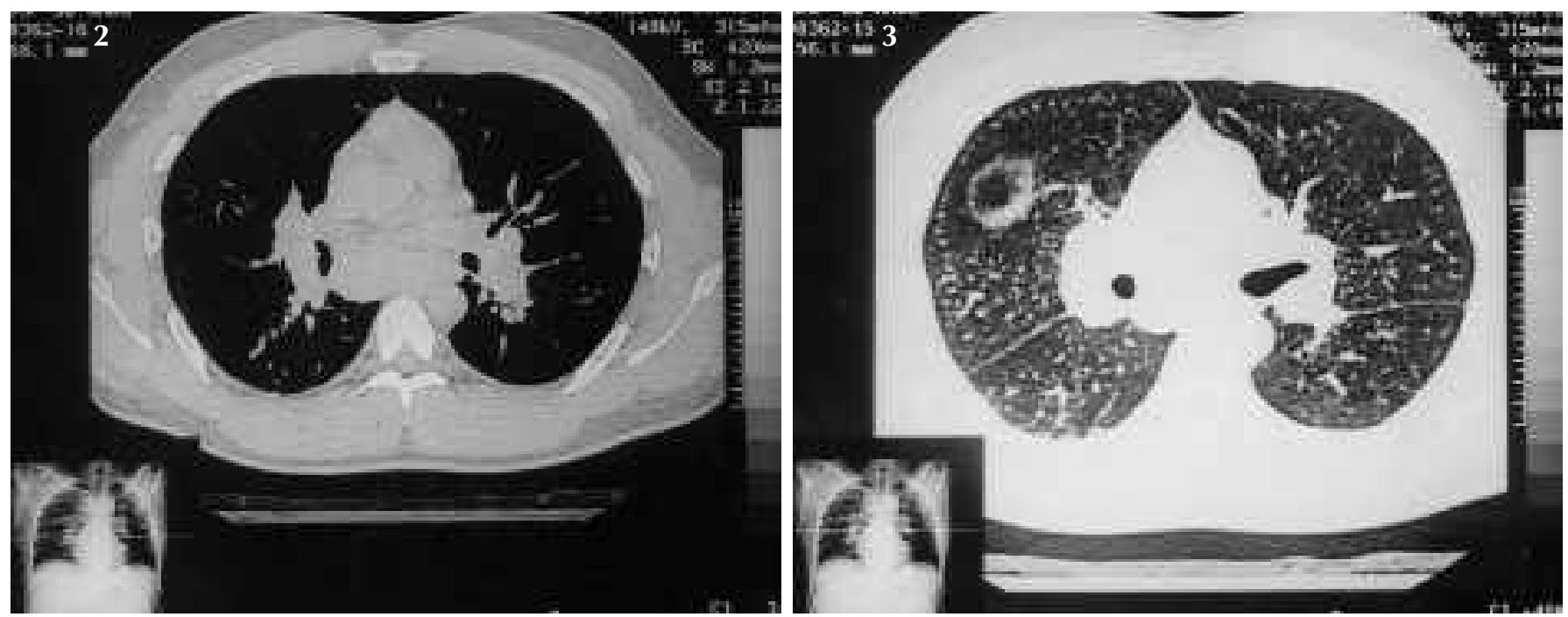

Figuras 2 e 3 - Tomografia de tórax. Linfonodomegalia hilar bilateral e lesão anular, com área central normal, em topografia de lobo superior direito.

Um dos achados mais úteis no reconhecimento da doença é a sua distribuição ao longo dos feixes broncovasculares, septos interlobulares e pleura, com tendência à bilateralidade e ao acometimento dos lobos superiores ${ }^{(2)}$. O padrão nodular é a anormalidade parenquimatosa mais freqüentemente detectada na TCAR desses pacientes $e$, de forma característica, a maioria dos nódulos são menores que $5 \mathrm{~mm}$ e apresentam margens irregulares ${ }^{(3)}$. Tais nódulos provavelmente representam grupos de granulomas intersticiais que coalesceram a ponto de serem visualizados pela TCAR ${ }^{(4)}$. Outras alterações também podem ser observadas na tomografia computadorizada da sarcoidose, incluindo espessamento da parede brônquica, opacificações em "vidro fosco", consolidações alveolares e pseudoalveolares, espessamento do septo interlobular, sinais de distorção da arquitetura pulmonar, honeycombing, bronquiectasias de tração e acometimento pleural(5).

Grande número de novos termos estão sendo continuadamente introduzidos na literatura para definir as anormalidades encontradas na tomografia computadorizada (em especial a técnica de alta resolução) das doenças pulmonares intersticiais. Recentemente, Marlow et al. descreveram um novo achado tomográfico na sarcoidose que consiste em lesões circulares, de diversos tamanhos, com áreas centrais de tecido aparentemente normal que eles chamaram de "anéis de fada"(6).

A expressão "anéis de fada" é bem conhecida na mitologia celta. Segundo a lenda, fadas apareciam nos bosques durante a noite e dançavam em pequenas círculos. Seus minúsculos pés movimentavam-se de forma esférica nas margens dos anéis. Já exaustas, elas iriam descansar. No dia seguinte, os viajantes, passando pelo lugar, viam apenas as pegadas em anéis ali deixadas. De forma aná- loga à radiologia, é plausivel a explicação da mitologia celta também à etiologia da sarcoidose. Já se passaram 124 anos desde a descrição inicial de Jonathan Hutchinson $^{(7)}$ e sua causa permanece um enigma apesar do rastro bem claro deixado pelo agente (ou agentes) nos pulmões.

O aspecto tomográfico acima descrito é atribuído pelos autores que primeiro o descreveram à resolução espontânea do processo granulomatoso central com o posterior desenvolvimento de novas lesões inflamatórias na periferia. O caso aqui descrito apresenta imagem semelhante àquela apresentada por Marlow et al.(6). O "anel de fada" é um provável sinal de doença e, dessa forma, é mais um achado que deve ser adicionado à extensa lista de lesões tomográficas encontradas na sarcoidose pulmonar.

\section{REFERÊNCIAS}

1. Hamper UM, Fishman EK, Khouri NF, Johns CJ, Wang KP, Siegelman SS. Typical and atypical CT manifestations of pulmonary sarcoidosis. J Comput Assist Tomogr 1986;10:928-936.

2. Müller NL, Kullnig P, Miller RR. The CT findings of pulmonary sarcoidosis: analysis of 25 patients. AJR Am J Roentgenol 1989;152:11791182.

3. Traill ZC, Maskell GF, Gleeson FV. High-resolution CT findings of pulmonary sarcoidosis. AJR Am J Roentgenol 1997;168:1557-1560.

4. Brito RR, Martinez JAB, Jasinowodolinski D, Ferreira RG. Imagens nodulares múltiplas em mulher jovem. J Pneumol 1997;23:47-49.

5. Hashimoto M, Watanabe O, Sato K, Endo K, Heianna J, Itoh I, Watarai J. The CT findings of pulmonary sarcoidosis. Tohoku J Exp Med 1996; 179:259-266.

6. Marlow TJ, Krapiva PI, Schabel SI, Judson MA. The "fairy ring" - a new radiographic finding in sarcoidosis. Chest 1999;115:275-276.

7. James DG. Descriptive definition and historic aspects of sarcoidosis. Clin Chest Med 1997;18:663-679. 


\title{
Imagem anular em tomografia computadorizada do tórax de alta resolução"
}

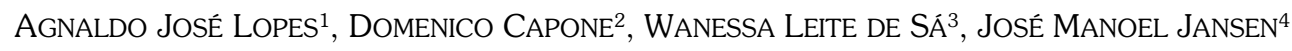

Descritores - Sarcoidose. Sarcoidose pulmonar. Tomografia computadorizada por raios X.

Key words - Sarcoidosis. Pulmonary sarcoidosis. X-ray computed tomography.

\section{APRESENTAÇÃO CLÍNICA}

Homem pardo, 32 anos, casado, instalador de aparelho de som, natural e residente no Rio de Janeiro, procurou atendimento médico queixando-se de tosse seca com um ano de evolução. Negava outros sintomas respiratórios, emagrecimento ou febre. Referia artralgia simétrica e cumulativa em cotovelos, punhos e tornozelos, sem sinais flogísticos associados. Negava tabagismo. Ao exame físico encontrava-se em bom estado geral e sem anormalidades.

Exames de imagem - A radiografia simples de tórax mostrava linfonodomegalia hilar bilateral (Figura 1). A tomografia computadorizada do tórax de alta resolução (TCAR) evidenciava aumento dos linfonodos hilares, micronódulos difusos e imagem anular de cerca de $4 \mathrm{~cm}$ de diâmetro, com parênquima normal de permeio, em lobo superior direito (Figuras 2 e 3).

Outros exames - Hematócrito $=51 \%$, hemoglobina $=$ $17 \mathrm{~g} / \mathrm{dl}$, leucócitos $=3.800 / \mathrm{mm}^{3}$. PPD não reator e dosagens de cálcio sérico e urinário normais. Exame de fundo de olho: edema cistóide em olho direito. A broncoscopia não evidenciava alterações de vias aéreas. A espirometria mostrava distúrbio ventilatório obstrutivo moderado. As medidas dos volumes pulmonares e da difusão do

\footnotetext{
Trabalho realizado no Hospital Universitário Pedro Ernesto.

1. Médico do Serviço de Pneumologia e Mestre em Pneumologia pela Universidade Federal Fluminense.

2. Professor Assistente do Serviço de Pneumologia do Hospital Universitário Pedro Ernesto e Radiologista do Hospital Geral de Bonsucesso.

3. Médica Estagiária do Serviço de Pneumologia.

4. Professor Titular e Chefe do Serviço de Pneumologia.

Endereço para correspondência - Agnaldo José Lopes, Rua José do Patrocínio, 290, Apto. 405 - 20560-160 - Rio de Janeiro, RJ. Tels. (21) 587-6357/587-6537/576-2532.
}

Recebido para publicação em 15/9/00. Aprovado, após revisão, em 12/3/01. monóxido de carbono foram normais. O exame anatomopatológico de fragmento retirado pela biópsia transbrônquica mostrou processo inflamatório crônico granulomatoso não caseoso com pesquisa negativa para micobactérias e fungos.

Diagnóstico - Sarcoidose gânglio-pulmonar.

\section{DISCUSSÃO}

Desde o seu surgimento, a tomografia computadorizada do tórax de alta resolução (TCAR) foi reconhecida como uma ferramenta importante na avaliação da sarcoidose pulmonar. Além de ver o pulmão com um detalhamento antes só possivel na observação microscópica da peça, pode-se estabelecer uma relação sinal (tomográfico) x significado (histológico), o que permitiu um grande avanço na busca diagnóstica e no acompanhamento das doenças pulmonares intersticiais.

A linfonodomegalia é tão comum quanto a doença do parênquima pulmonar em pacientes com sarcoidose. A tomografia computadorizada é superior à radiografia de tórax para definir a extensão do envolvimento ganglionar e demonstrar a sua presença em localizações atípicas tais como o mediastino anterior, axilas, a região subcarinal e a cadeia mamária interna. $\mathrm{O}$ acometimento linfonodal geralmente é simétrico e bilateral, e os gânglios linfáticos podem apresentar calcificações, inclusive com aspecto em "casca de ovo"(1).

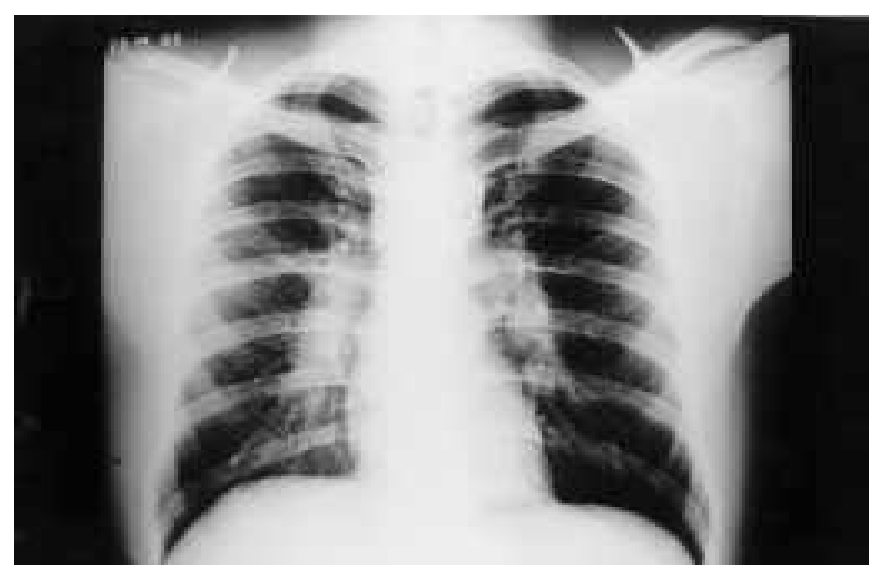

Figura 1 - Radiografia de tórax mostrando linfonodomegalia hilar bilateral e ausência de anormalidades no parênquima pulmonar. 

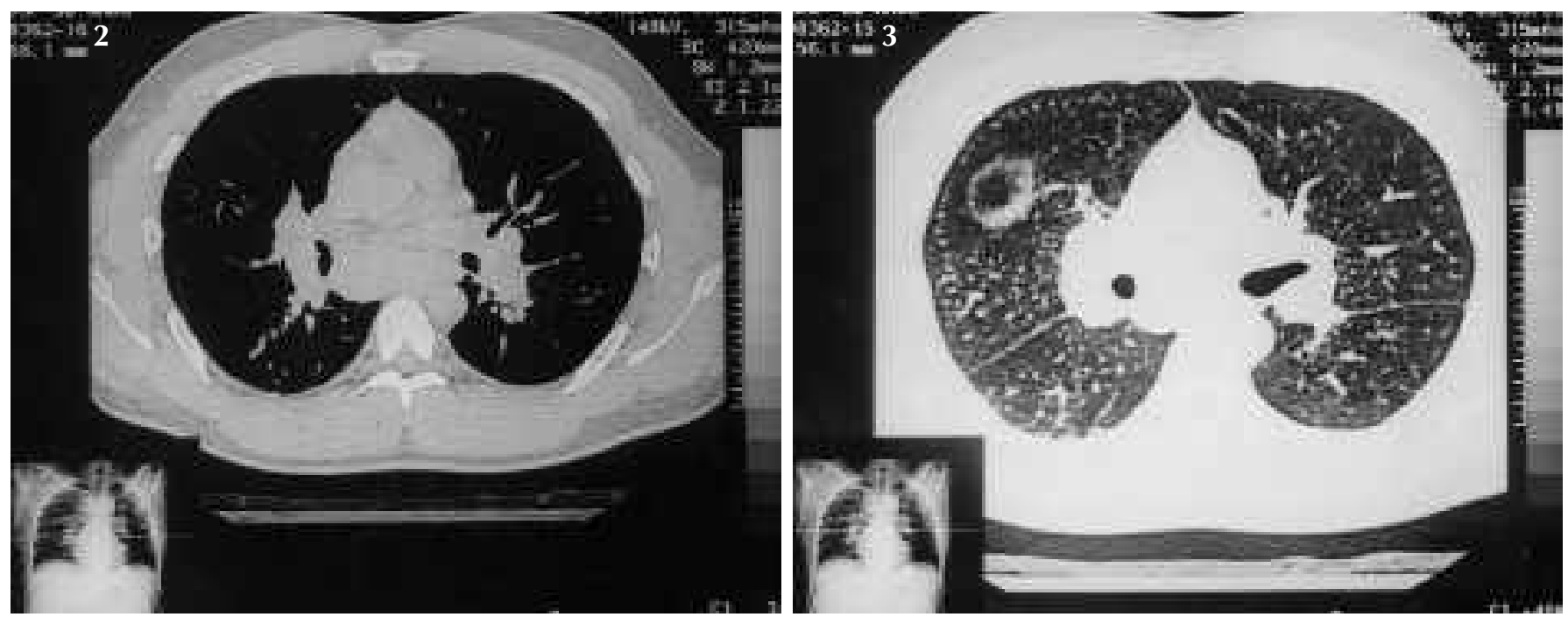

Figuras 2 e 3 - Tomografia de tórax. Linfonodomegalia hilar bilateral e lesão anular, com área central normal, em topografia de lobo superior direito.

Um dos achados mais úteis no reconhecimento da doença é a sua distribuição ao longo dos feixes broncovasculares, septos interlobulares e pleura, com tendência à bilateralidade e ao acometimento dos lobos superiores ${ }^{(2)}$. O padrão nodular é a anormalidade parenquimatosa mais freqüentemente detectada na TCAR desses pacientes $e$, de forma característica, a maioria dos nódulos são menores que $5 \mathrm{~mm}$ e apresentam margens irregulares ${ }^{(3)}$. Tais nódulos provavelmente representam grupos de granulomas intersticiais que coalesceram a ponto de serem visualizados pela TCAR ${ }^{(4)}$. Outras alterações também podem ser observadas na tomografia computadorizada da sarcoidose, incluindo espessamento da parede brônquica, opacificações em "vidro fosco", consolidações alveolares e pseudoalveolares, espessamento do septo interlobular, sinais de distorção da arquitetura pulmonar, honeycombing, bronquiectasias de tração e acometimento pleural(5).

Grande número de novos termos estão sendo continuadamente introduzidos na literatura para definir as anormalidades encontradas na tomografia computadorizada (em especial a técnica de alta resolução) das doenças pulmonares intersticiais. Recentemente, Marlow et al. descreveram um novo achado tomográfico na sarcoidose que consiste em lesões circulares, de diversos tamanhos, com áreas centrais de tecido aparentemente normal que eles chamaram de "anéis de fada"(6).

A expressão "anéis de fada" é bem conhecida na mitologia celta. Segundo a lenda, fadas apareciam nos bosques durante a noite e dançavam em pequenas círculos. Seus minúsculos pés movimentavam-se de forma esférica nas margens dos anéis. Já exaustas, elas iriam descansar. No dia seguinte, os viajantes, passando pelo lugar, viam apenas as pegadas em anéis ali deixadas. De forma aná- loga à radiologia, é plausivel a explicação da mitologia celta também à etiologia da sarcoidose. Já se passaram 124 anos desde a descrição inicial de Jonathan Hutchinson $^{(7)}$ e sua causa permanece um enigma apesar do rastro bem claro deixado pelo agente (ou agentes) nos pulmões.

O aspecto tomográfico acima descrito é atribuído pelos autores que primeiro o descreveram à resolução espontânea do processo granulomatoso central com o posterior desenvolvimento de novas lesões inflamatórias na periferia. O caso aqui descrito apresenta imagem semelhante àquela apresentada por Marlow et al.(6). O "anel de fada" é um provável sinal de doença e, dessa forma, é mais um achado que deve ser adicionado à extensa lista de lesões tomográficas encontradas na sarcoidose pulmonar.

\section{REFERÊNCIAS}

1. Hamper UM, Fishman EK, Khouri NF, Johns CJ, Wang KP, Siegelman SS. Typical and atypical CT manifestations of pulmonary sarcoidosis. J Comput Assist Tomogr 1986;10:928-936.

2. Müller NL, Kullnig P, Miller RR. The CT findings of pulmonary sarcoidosis: analysis of 25 patients. AJR Am J Roentgenol 1989;152:11791182.

3. Traill ZC, Maskell GF, Gleeson FV. High-resolution CT findings of pulmonary sarcoidosis. AJR Am J Roentgenol 1997;168:1557-1560.

4. Brito RR, Martinez JAB, Jasinowodolinski D, Ferreira RG. Imagens nodulares múltiplas em mulher jovem. J Pneumol 1997;23:47-49.

5. Hashimoto M, Watanabe O, Sato K, Endo K, Heianna J, Itoh I, Watarai J. The CT findings of pulmonary sarcoidosis. Tohoku J Exp Med 1996; 179:259-266.

6. Marlow TJ, Krapiva PI, Schabel SI, Judson MA. The "fairy ring" - a new radiographic finding in sarcoidosis. Chest 1999;115:275-276.

7. James DG. Descriptive definition and historic aspects of sarcoidosis. Clin Chest Med 1997;18:663-679. 